\title{
Vocalização como indicativo do bem-estar de suínos submetidos a situações de estresse
}

\author{
[Vocalization as a welfare indicative for pigs subjected to stress situations] \\ M. Moi, I.A. Nääs, F.R. Caldara, I.C.L.A. Paz, R.G. Garcia, \\ A.F.S. Cordeiro, L.O. Seno
}

Universidade Federal da Grande Dourados - Itahum, MS

\begin{abstract}
RESUMO
Objetivou-se com este trabalho estimar o bem-estar de suínos a partir de registros de sua vocalização, durante alojamento em granja comercial. Foram utilizados 150 suínos machos castrados, distribuídos aleatoriamente em cinco baias. Os suínos foram submetidos a diferentes situações de estresse: sede (sem acesso a água), fome (sem acesso ao alimento), estresse térmico (Índice de temperatura e umidade - ITU >74). Para o tratamento controle, os animais foram mantidos em situação de conforto, com acesso a alimento e água, e ambiente com ITU <70. Foram registrados os sinais acústicos a cada 30 minutos, durante período ininterrupto de três horas, totalizando seis coletas para cada situação de estresse. Os sinais foram digitalizados a uma frequência de até $44.100 \mathrm{~Hz}$, por um período de 3 minutos. Posteriormente, os áudios foram analisados pelo software Praat ${ }^{\circledR}$ 5.1.19. Os atributos gerados a partir desse software foram a energia do sinal $\left(\mathrm{Pa}^{2} * \mathrm{~s}\right)$, amplitude máxima e amplitude mínima $(\mathrm{Pa})$, a frequência de picht $(\mathrm{Hz})$, a intensidade sonora $(\mathrm{dB})$ e quatro níveis de formantes (F1; F2; F3 e F4), também chamados de harmônicas $(\mathrm{Hz})$. Verificou-se que, dependendo do estímulo estressor e de sua duração, os atributos acústicos energia e intensidade do sinal, frequência de pitch e as formantes 2 e 4 apresentaram diferenciação. Os atributos sonoros da vocalização de suínos variam de maneira distinta em função do tipo e duração do estímulo estressante, funcionando, quando associados, como ferramenta eficiente para quantificar o grau de estresse dos animais.
\end{abstract}

Palavras-chave: ambiência, estresse, suinocultura, zootecnia de precisão

\begin{abstract}
This work aimed to estimate the welfare of pigs using their vocalization records during rearing in a commercial swine farm. A total of 150 barrow pigs were randomly distributed into five pens. Pigs were exposed to different stressful situations: Thirst (no access to water), hunger (no access to the food), thermal stress (Temperature and Humidity Index - THI >74). For the control treatment, the animals were kept in a situation of comfort, with full access to food and water, and environment THI <70. Acoustic signals were recorded every 30 minutes during a continuous period of three hours, totaling six samples for each stress exposure. The signals were digitized at a frequency of up to 44,100 $\mathrm{Hz}$, for a period of 3 minutes. Later the audios were analyzed using the Praat ${ }^{\circledR}$ 5.1.19 software. The attributes generated from this software were the signal energy $\left(\mathrm{Pa}^{2} * s\right)$, the maximum amplitude and the minimum amplitude $(\mathrm{Pa})$, the frequency of pitch $(\mathrm{Hz})$, the sound intensity $(\mathrm{dB})$ and four levels of formants $(F 1, F 2, F 3$ and F4), also called harmonics $(\mathrm{Hz})$. It was found that depending on the acoustic attributes, the stressor stimuli and its duration indicate energy and signal strength, frequency of pitch and formants 2 and 4 showed differentiation. The sound attributes of the pig vocalization varied in different ways depending on the type and duration of the stressful exposure, functioning as an efficient tool to quantify the degree of animal degree.
\end{abstract}

Keywords: ambience, stress, pig farming, precision livestock production

Recebido em 23 de julho de 2013

Aceito em 16 de março de 2015

E-mail: mar_moi@hotmail.com 


\section{INTRODUÇÃO}

O aumento da produção brasileira de carne suína foi de 2.621,3 mil toneladas e exportação de 508 mil toneladas em 2004, para 3.428,6 mil toneladas com exportações da ordem de $517 \mathrm{mil}$ toneladas em 2013 (Associação..., 2015). Esse cenário, aliado ao fato de os mercados importadores estarem mais exigentes quanto às condições em que os animais são criados e o impacto da produção sobre o meio ambiente, determina a necessidade de maior atenção com o bem-estar animal.

O estresse é um dos principais parâmetros de avaliação do bem-estar animal (Goymann et al., 2003). As medidas fisiológicas associadas ao estresse têm sido usadas baseadas em que, se o estresse aumenta, o bem-estar diminui. Já os indicadores comportamentais são baseados especialmente na ocorrência de comportamentos anormais, e daqueles que se afastam do comportamento no ambiente natural (Hötzel e Machado Filho, 2004). Em situações de estresse ou conflito, os suínos são capazes de expressar diferentes comportamentos individuais para conseguir lidar com essas situações. A vocalização é uma das ferramentas que utilizam para se expressar e pode ser percebida e tomada como parâmetro mesmo nas primeiras semanas de suas vidas (Hessing et al., 1993). As vocalizações emitidas em situações de estresse podem servir como um indicador de qualidade de vida dos animais submetidos a diferentes estímulos estressantes e como uma avaliação instantânea do estado do animal (Düpjan et al., 2008).

Objetivou-se com esta pesquisa avaliar a possibilidade de distinguir diferentes estímulos estressantes (fome, sede e estresse térmico), bem como a intensidade do estresse de suínos por meio de sua vocalização.

\section{MATERIAL E MÉTODOS}

O experimento foi realizado no mês de abril de 2012, em uma granja comercial situada no noroeste do Estado do Rio Grande do Sul, localização (latitude $-27^{\circ} 55^{\prime}$ 23"S, longitude $53^{\circ} 02^{\prime} 21^{\prime \prime O}$ ) e altitude média de $385 \mathrm{~m}$. O clima da região, de acordo com a classificação de Köppen, é subtropical úmido.
Foram utilizados 150 suínos machos castrados cirurgicamente, da linhagem comercial Dalland®, em fase de crescimento (100 dias de idade), distribuídos aleatoriamente em cinco baias. Os suínos foram alojados em galpão com orientação no sentido leste-oeste, pé-direito de 3,0 metros, muretas laterais de 1 metro de altura, cortinas laterais e cobertura de telhas de amianto (6mm). O galpão utilizado possui 10 baias com $30 \mathrm{~m}^{2}$ de área, sendo alocados 30 animais em cada, em densidade de $1 \mathrm{~m}^{2}$ por animal. As baias são compostas por piso de concreto com lâmina d'água de $5 \mathrm{~cm}$ e equipadas com bebedouros do tipo "chupeta", dispostos no fundo das baias (três por baia), e comedouro do tipo automático. Nas laterais externas dos galpões, incide uma linha de árvores, à distância de 3 metros da mureta lateral, com objetivo de propiciar sombra na parte superior do galpão (telhado).

Registraram-se os dados ambientais (temperatura e umidade relativa) usando-se um termohigrômetro digital, com registro a cada 30 minutos. Utilizando-se os valores médios de temperatura e umidade relativa do ar, foram calculados as temperaturas de bulbo seco (TBS) e bulbo úmido (TBU) do ar por meio do programa Psicrom ${ }^{\circledR} \quad$ (Roriz, 2003) e posteriormente os Índices de Temperatura e Umidade (ITU) utilizando-se a Equação: ITU = 0,45 Tbu + 1,35 Tbs + 32 (Roller e Goldman, 1969). Em dias alternados, os animais foram submetidos às diferentes situações de estresse: sede (sem acesso a água), fome (sem acesso a alimento), estresse térmico (ITU superior a 74). Para o tratamento controle, os animais foram mantidos em situação de conforto (animais com acesso a alimento e água e ambiente com ITU abaixo de 73). Para avaliação das situações de estresse por fome e sede, os animais permaneceram por 11 horas em jejum, durante o período noturno, e, a partir desse período, iniciou-se a coleta dos dados. A avaliação da situação de estresse térmico se iniciou a partir das 13 h00min, quando a temperatura do ITU do galpão estava acima de 74. Registraram-se os sinais acústicos a cada 30 minutos, durante período ininterrupto de três horas, totalizando seis coletas para cada situação de estresse. O registro dos sinais acústicos foi realizado com o auxílio de um microfone unidirecional YOGA ${ }^{\circledR} \mathrm{e}$ gravador digital Marantz ${ }^{\circledR}$ PMD 660. O microfone foi posicionado a $1 \mathrm{~m}$ de altura dos suínos, disposto no centro de cada baia, sendo 
este acoplado ao gravador. Os sinais foram digitalizados a uma frequência de até $44.100 \mathrm{~Hz}$, por um período de 3 minutos.

Após a realização das coletas, os sons foram descarregados em um computador. Cada baia proporcionou o registro de uma faixa sequencial de "gritos e grunhidos" (vocalizações). Posteriormente os áudios foram analisados pelo software Praat ${ }^{\circledR}$ 5.1.19, sendo extraídos os parâmetros acústicos pela aplicação da Transformada de Fourier, gerando um espectro sonoro. Os atributos gerados a partir desse software foram a energia do sinal $\left(\mathrm{Pa}^{2 *} \mathrm{~s}\right)$, amplitude máxima e amplitude mínima $(\mathrm{Pa})$, a frequência de picht $(\mathrm{Hz})$, a intensidade sonora
(dB) e quatro níveis de formantes $(\mathrm{F} 1 ; \mathrm{F} 2 ; \mathrm{F} 3 \mathrm{e}$ F4), também chamados de harmônicas (Hz).

A análise estatística foi realizada com auxílio do pacote computacional SAS 9.2 (2000), sendo realizados testes de Normalidade e Homocedasticidade dos dados e posteriormente submetidos às análises de Variância e teste de Tukey.

\section{RESULTADOS E DISCUSSÃO}

No sonograma das vocalizações, encontram-se as variáveis identificadas durante as gravações (Fig. 1).

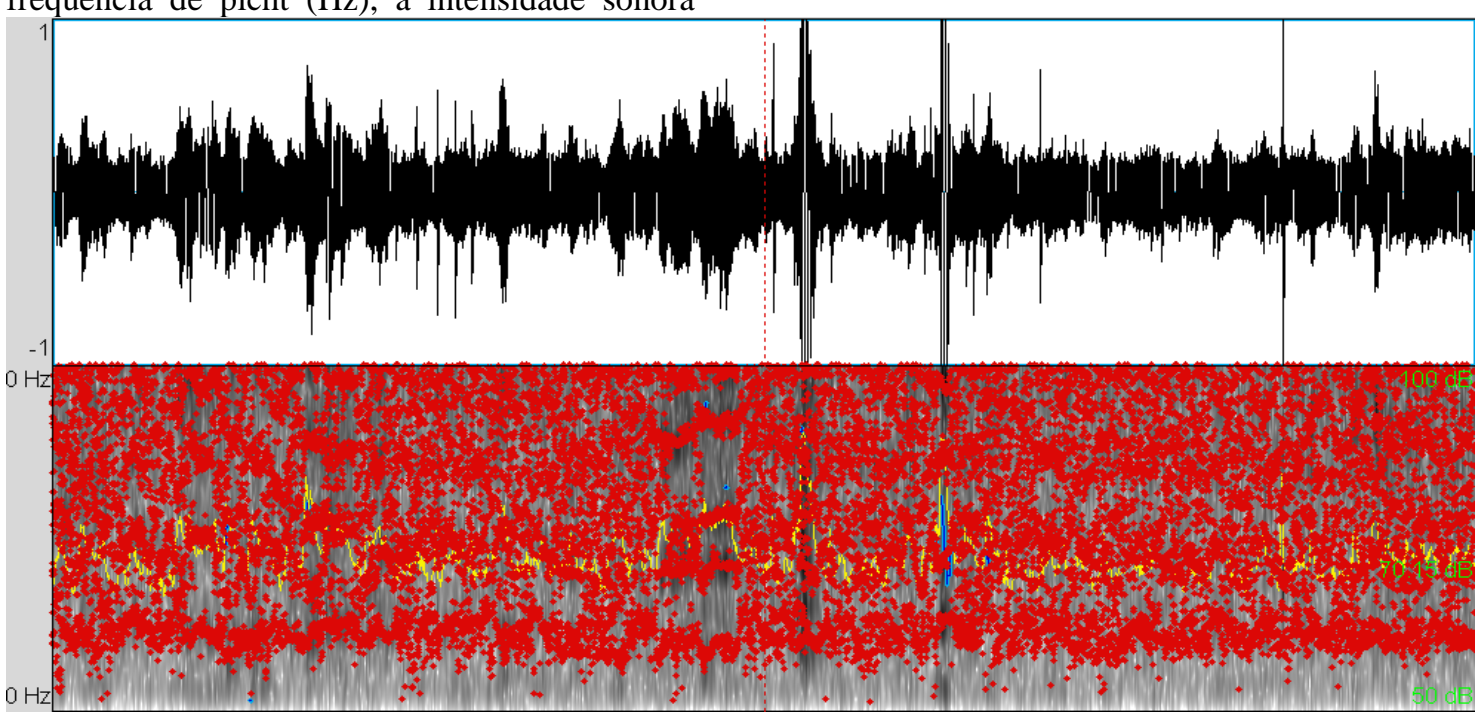

Figura 1. Sonograma de vocalizações de suínos. Pontilhados vermelhos são as formantes; linha em amarelo, a intensidade do som, e pontilhados em azul, a frequência de pitch.

Considerando-se conjuntamente os fatores climáticos temperatura e umidade relativa do ar para determinação do índice de temperatura e umidade (ITU), observou-se que, durante os dias de avaliação dos estímulos de fome e sede, bem como na situação de bem-estar (sem estresse), os animais encontravam-se sob conforto térmico, não tendo, dessa forma, o efeito do calor interferindo nas demais avaliações (Tab. 1). Segundo Barbari et al. (2007), suínos encontramse em condição de conforto térmico quando o ITU for menor ou igual a 74. Valores de ITU maiores ou iguais a 75 indicam condição de alerta e, acima de 79, condição de perigo.

Tabela 1. Médias e desvios padrão de temperatura $\left(\mathrm{T}^{\circ} \mathrm{C}\right)$; umidade relativa do ar (UR\%) e índice de temperatura e umidade (ITU) observados nos dias de avaliações da vocalização de suínos submetidos a diferentes estímulos estressantes

\begin{tabular}{ccccc}
\hline \multicolumn{4}{c}{ Condição } \\
\hline Parâmetros & Sede & Estresse Térmico & Fome & BEA \\
\hline $\mathrm{T}^{\circ} \mathrm{C}$ & $15,23 \pm 0,85$ & $31,82 \pm 1,78$ & $15,28 \pm 0,68$ & $22,43 \pm 1,34$ \\
UR\% & $78,05 \pm 3,56$ & $34,08 \pm 4,25$ & $81,90 \pm 2,17$ & $30,50 \pm 2,13$ \\
ITU & $58,38 \pm 1,44$ & $83,93 \pm 2,66$ & $58,67 \pm 1,22$ & $67,90 \pm 2,12$ \\
\hline
\end{tabular}


As médias dos atributos acústicos gerados pelas vocalizações foram comparadas entre os estímulos estressantes (sede, fome, estresse térmico) e condição de conforto (Tab. 2).

Foram avaliadas as vocalizações dos suínos em função do tempo de duração do estímulo estressante (Tab. 3). Exceto para as formantes $1 \mathrm{e}$ 3 , todos os atributos sonoros avaliados apresentaram diferença significativa entre os estímulos aos quais os animais foram submetidos. Entretanto os mesmos se comportaram de maneira distinta (Tab. 2).

Tabela 2. Média geral dos atributos acústicos: energia do sinal (ES), amplitude máxima (AA), amplitude mínima (AI), intensidade do som (IS), frequência de pitch (FP) e formantes 1, 2, 3 e 4 (F1, F2, F3 e F4) de vocalizações de suínos machos submetidos a estresse por sede, fome, calor, e em condição de bemestar (BEA)

\begin{tabular}{cccccc}
\hline & \multicolumn{5}{c}{ Tratamento } \\
\hline Atributos & Sede & Calor & Fome & BEA & CV\% \\
\hline ES & $5,31 \mathrm{c}$ & $17,35 \mathrm{a}$ & $12,02 \mathrm{~b}$ & $7,48 \mathrm{c}$ & 22,13 \\
AA & $1,71 \mathrm{~b}$ & $1,93 \mathrm{a}$ & $1,90 \mathrm{ab}$ & $1,75 \mathrm{ab}$ & 6,10 \\
AI & $1,64 \mathrm{~b}$ & $1,82 \mathrm{a}$ & $1,84 \mathrm{a}$ & $1,68 \mathrm{ab}$ & 5,61 \\
IS & $77,75 \mathrm{~b}$ & $83,47 \mathrm{a}$ & $81,78 \mathrm{a}$ & $75,76 \mathrm{~b}$ & 2,03 \\
FP & $267,96 \mathrm{~b}$ & $325,40 \mathrm{a}$ & $185,92 \mathrm{c}$ & $310,13 \mathrm{a}$ & 7,95 \\
F1 & $1058,46 \mathrm{a}$ & $1084,33 \mathrm{a}$ & $1068,83 \mathrm{a}$ & $1082,28 \mathrm{a}$ & 1,57 \\
F2 & $2116,46 \mathrm{ab}$ & $2151,69 \mathrm{a}$ & $2152,02 \mathrm{a}$ & $2094,52 \mathrm{~b}$ & 0,97 \\
F3 & $3210,95 \mathrm{a}$ & $3249,31 \mathrm{a}$ & $3250,97 \mathrm{a}$ & $3221,35 \mathrm{a}$ & 0,81 \\
F4 & $4232,47 \mathrm{ab}$ & $4269,43 \mathrm{ab}$ & $4275,74 \mathrm{a}$ & $4218,20 \mathrm{~b}$ & 0,70 \\
\hline
\end{tabular}

Nas linhas, médias seguidas por letras diferentes diferem estatisticamente entre si pelo Teste Tukey $(\mathrm{P}>0,05)$.

A energia do sinal não permitiu a diferenciação da condição de estresse por sede da situação de conforto. Entretanto, ambas foram distintas das demais condições (calor e fome), que, por sua vez, também foram diferentes entre si. A energia do sinal proveniente da vocalização de suínos é significativamente maior em animais submetidos ao estresse por calor, seguido da condição de fome. Portanto, o atributo energia do sinal pode ser um bom indicativo de que os animais estão submetidos à condição de estresse térmico ou de fome. Não houve efeito regular da duração do estresse sobre a energia do sinal da vocalização nas diferentes situações (Tab. 3 e Fig. 2).

Contradizendo os resultados observados, Cordeiro et al. (2009) verificaram que a energia do sinal aumenta em função do estresse a que o animal é submetido. Marx et al. (2003), avaliando suínos durante a castração, verificaram que as vocalizações associadas com a dor podem ser identificadas e caracterizadas por meio da energia do sinal. Comparando a vocalização de animais castrados sem anestesia e com anestesia local, Cordeiro (2012) observou que não houve diferença da energia emitida na vocalização dos leitões nessas duas situações.

As amplitudes máxima e mínima das vocalizações não foram distintas entre suínos submetidos à condição de calor, fome ou bemestar. Apenas sede e estresse térmico puderam ser diferenciados entre si, sendo as amplitudes maiores para animais submetidos ao estresse por calor. Dessa forma, a amplitude máxima não foi considerada um bom indicativo para diferenciação de estresse. A duração do estresse não influenciou as amplitudes máxima e mínima (Tab. 3, Fig. 3 e 4).

A intensidade do sinal não permitiu a diferenciação da condição de estresse por sede da situação de conforto. Entretanto, ambas foram distintas das demais condições (calor e fome), que, por sua vez, não foram diferentes entre si. A intensidade do sinal proveniente da vocalização de suínos é maior em animais submetidos ao estresse por calor e por fome do que pela sede ou em situação de conforto. Dessa forma, quando associado ao atributo energia do sinal, pode ser um bom indicativo de condições de estresse térmico ou de fome. A intensidade do som apresentou comportamento constante ao longo do tempo (Tab. 3 e Fig. 5). Trabalhando com suínos em diferentes situações de estresse (estresse por calor, sede, frio, dor e condição de bem-estar), Cordeiro (2012) observou valores de intensidade do sinal (entre 72 e $76 \mathrm{~dB}$ ) próximos aos encontrados na presente pesquisa. 
Vocalização como indicativo...

Tabela 3. Atributos de vocalizações de suínos em função da duração do estímulo estressante (Estresse por sede, estresse térmico, estresse por fome e condição de bem-estar animal - BEA)

\begin{tabular}{|c|c|c|c|c|c|c|c|c|}
\hline \multirow{2}{*}{\multicolumn{2}{|c|}{ Atributos }} & \multicolumn{7}{|c|}{ Períodos } \\
\hline & & 1 & 2 & 3 & 4 & 5 & 6 & $\mathrm{CV} \%$ \\
\hline \multirow{9}{*}{ 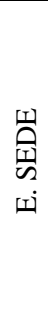 } & ES & $10,70 \mathrm{a}$ & $3,02 \mathrm{~b}$ & $5,91 \mathrm{~b}$ & $3,02 \mathrm{~b}$ & $4,11 b$ & $5,07 \mathrm{~b}$ & 38,25 \\
\hline & AA & 1,84 & 1,55 & 1,83 & 1,48 & 1,75 & 1,79 & 13,54 \\
\hline & AI & 1,70 & 1,53 & 1,72 & 1,51 & 1,68 & 1,67 & 11,02 \\
\hline & IS & $80,95 \mathrm{a}$ & $75,99 b$ & $78,51 \mathrm{ab}$ & $75,91 \mathrm{~b}$ & $77,10 \mathrm{~b}$ & $78,03 \mathrm{ab}$ & 2,33 \\
\hline & FP & 312,51 & 245,29 & 286,67 & 226,24 & 239,75 & 288,30 & 18,61 \\
\hline & $\mathrm{F} 1$ & 1047,70 & 1051,30 & 1059,59 & 1052,49 & 1074,16 & 1065,50 & 1,99 \\
\hline & $\mathrm{F} 2$ & $2034,47 b$ & $2116,18 \mathrm{ab}$ & $2131,79 a$ & $2147,30 \mathrm{a}$ & $2142,63 a$ & $2126,38 \mathrm{a}$ & 2,16 \\
\hline & F3 & $3095,50 b$ & $3202,79 a$ & $3251,57 \mathrm{a}$ & $3254,08 \mathrm{a}$ & $3233,03 \mathrm{a}$ & $3228,73 a$ & 0,94 \\
\hline & $\mathrm{F} 4$ & $4133,80 \mathrm{~b}$ & $4232,75 \mathrm{a}$ & $4244,72 \mathrm{a}$ & $4274,06 \mathrm{a}$ & $4266,78 \mathrm{a}$ & $4242,73 \mathrm{a}$ & 0,71 \\
\hline \multirow{9}{*}{ 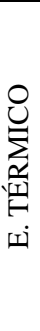 } & ES & 19,85 & 18,05 & 15,95 & 15,76 & 19,27 & 15,21 & 32,88 \\
\hline & AA & 1,92 & 1,94 & 1,98 & 1,81 & 2,04 & 1,92 & 8,32 \\
\hline & AI & 1,73 & 1,87 & 1,93 & 1,72 & 1,90 & 1,79 & 8,33 \\
\hline & IS & 84,17 & 83,46 & 83,13 & 83,07 & 83,94 & 83,06 & 1,71 \\
\hline & FP & 386,65 & 292,79 & 301,27 & 332,64 & 313,86 & 325,20 & 19,60 \\
\hline & $\mathrm{F} 1$ & $1120,81 \mathrm{a}$ & $1096,30 \mathrm{ab}$ & $1082,65 \mathrm{ab}$ & $1081,45 \mathrm{ab}$ & $1058,48 b$ & $1066,29 b$ & 2,07 \\
\hline & $\mathrm{F} 2$ & 2168,43 & 2156,45 & 2152,67 & 2146,78 & 2143,38 & 2142,45 & 1,57 \\
\hline & F3 & 3268,06 & 3249,41 & 3255,24 & 3246,63 & 3244,25 & 3232,25 & 1,01 \\
\hline & F4 & 4292,57 & 4261,30 & 4269,42 & 4270,13 & 4264,44 & 4258,71 & 0,71 \\
\hline \multirow{9}{*}{ 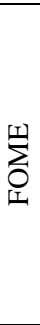 } & ES & $15,21 \mathrm{a}$ & $12,14 \mathrm{ab}$ & $7,60 \mathrm{~b}$ & $12,27 \mathrm{ab}$ & $10,46 a b$ & $14,43 \mathrm{ab}$ & 29,26 \\
\hline & AA & 2,04 & 1,95 & 1,73 & 1,87 & 1,84 & 1,98 & 9,15 \\
\hline & $\mathrm{AI}$ & $1,98 \mathrm{a}$ & $1,84 \mathrm{ab}$ & $1,58 b$ & $1,86 a b$ & $1,87 \mathrm{ab}$ & $1,92 \mathrm{ab}$ & 9,87 \\
\hline & IS & $83,10 \mathrm{a}$ & $81,95 \mathrm{ab}$ & $79,80 \mathrm{~b}$ & $82,14 \mathrm{ab}$ & $81,13 \mathrm{ab}$ & $82,54 \mathrm{ab}$ & 1,94 \\
\hline & FP & 204,46 & 197,92 & 178,05 & 151,09 & 200,39 & 183,61 & 17,89 \\
\hline & $\mathrm{F} 1$ & 1062,69 & 1078,11 & 1070,75 & 1055,54 & 1072,11 & 1073,76 & 2,53 \\
\hline & $\mathrm{F} 2$ & $2118,87 b$ & $2158,29 \mathrm{ab}$ & $2203,96 a$ & $2164,35 \mathrm{ab}$ & $2125,75 b$ & $2140,88 \mathrm{ab}$ & 1,56 \\
\hline & F3 & $3195,64 b$ & $3257,77 \mathrm{ab}$ & $3305,29 a$ & $3266,57 \mathrm{ab}$ & $3225,11 b$ & $3255,43 \mathrm{ab}$ & 1,14 \\
\hline & $\mathrm{F} 4$ & $4235,00 \mathrm{~b}$ & $4286,12 \mathrm{ab}$ & $4318,22 \mathrm{a}$ & $4293,64 a b$ & $4250,05 \mathrm{~b}$ & $4271,43 \mathrm{ab}$ & 0,75 \\
\hline \multirow{9}{*}{$\underset{\pi}{\pi}$} & ES & $9,60 \mathrm{ab}$ & $1034 \mathrm{a}$ & $11,99 \mathrm{a}$ & $4,15 \mathrm{ab}$ & $0,64 \mathrm{~b}$ & $8,12 \mathrm{ab}$ & 62,72 \\
\hline & AA & $1,94 \mathrm{a}$ & $1,89 \mathrm{a}$ & $1,97 \mathrm{a}$ & $1,52 \mathrm{ab}$ & $1,31 b$ & $1,88 \mathrm{a}$ & 14,81 \\
\hline & AI & $1,85 \mathrm{a}$ & $1,77 \mathrm{ab}$ & $1,88 \mathrm{a}$ & $1,46 a b$ & $1,33 b$ & $1,78 \mathrm{ab}$ & 14,36 \\
\hline & IS & $80,01 \mathrm{a}$ & $81,00 \mathrm{a}$ & $79,22 \mathrm{a}$ & $72,62 \mathrm{ab}$ & $65,22 \mathrm{~b}$ & $76,47 \mathrm{a}$ & 7,32 \\
\hline & FP & $337,93 \mathrm{a}$ & $338,63 a$ & $313,23 \mathrm{ab}$ & $298,50 \mathrm{ab}$ & $268,70 b$ & $303,76 \mathrm{ab}$ & 9,46 \\
\hline & $\mathrm{F} 1$ & 1076,18 & 1091,26 & 1094,28 & 1073,18 & 1082,70 & 1076,09 & 2,33 \\
\hline & F2 & 2072,23 & 2103,68 & 2053,66 & 2117,56 & 2114,47 & 2105,56 & 3,28 \\
\hline & F3 & 3214,87 & 3198,71 & 3284,14 & 3200,88 & 3226,75 & 3202,73 & 2,77 \\
\hline & $\mathrm{F} 4$ & 4184,76 & 4214,46 & 4234,75 & 4219,01 & 4237,74 & 4218,47 & 2,83 \\
\hline
\end{tabular}

Nas linhas, médias seguidas por letras diferentes diferem estatisticamente entre si pelo Teste Tukey $(\mathrm{P}>0,05)$. ES: energia do sinal; AA: amplitude máxima; AI: amplitude mínima; IS: intensidade do sinal; FP: frequência de pitch; F1: formante 1; F2: formante 2; F3: formante 3; F4: formante 4. Períodos 1, 2, 3, 4, 5 e 6 correspondem a cada 30 minutos de intervalo de avaliação.

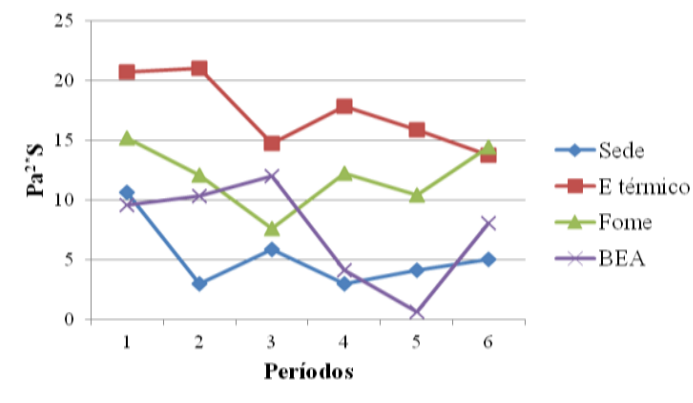

Figura 2. Energia do sinal de vocalizações de suínos submetidos a diferentes estímulos estressantes.

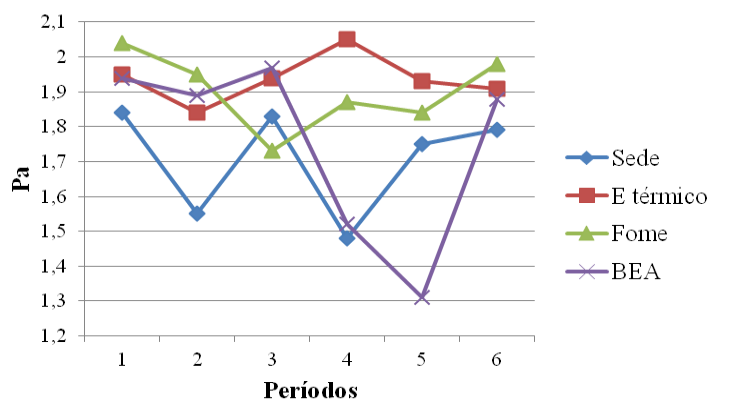

Figura 3. Amplitude máxima de vocalizações de suínos submetidos a diferentes estímulos estressantes. 


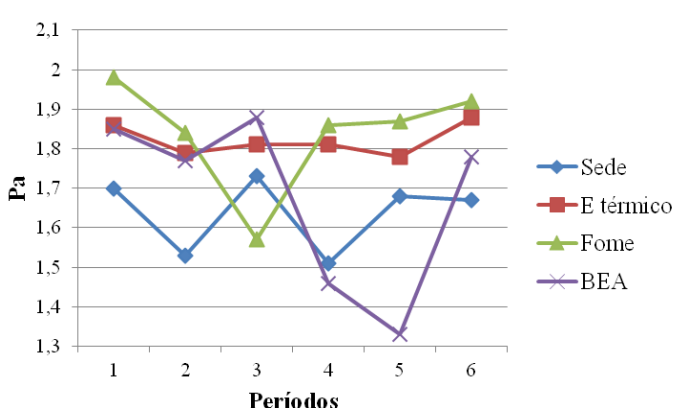

Figura 4. Amplitude mínima de vocalizações de suínos submetidos a diferentes estímulos estressantes.

Avaliando leitões em fase de maternidade, Risi (2010) concluiu que leitões sadios emitem gritos com maior intensidade $(79,76 \mathrm{~dB})$ do que animais artríticos $(78,15 \mathrm{~dB})$. Uma das explicações para a ocorrência desse fato deve-se ao melhor desempenho dos animais sadios em estimular a expulsão de ar pelas vias aéreas, promovendo a vibração das cordas vocais.

Buscando estimar o nível de dor em suínos pela sua vocalização, Cordeiro (2012) proporcionou diferentes condições de estresse por dor (marcação, caudectomia e castração) em leitões em fase de maternidade. A autora concluiu que a intensidade do som aumenta sucessivamente de normal $(70,41 \mathrm{~dB})$ para marcação $(77,64 \mathrm{~dB})$, depois para caudectomia $(88,31 \mathrm{~dB})$ e castração $(87,39 \mathrm{~dB})$, sugerindo que a intensidade do som vocal do suíno acompanha o aumento do nível de dor.

Desse modo, com base nos resultados observados na presente pesquisa, pode-se inferir

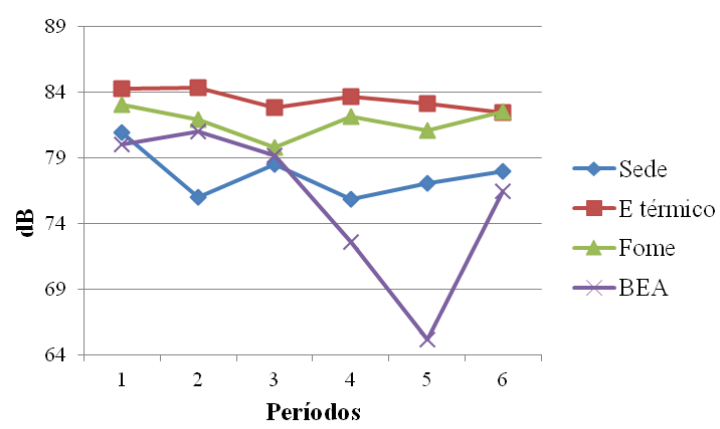

Figura 5. Intensidade do som de vocalizações de suínos submetidos a diferentes estímulos estressantes.

que suínos submetidos a fatores estressantes, mesmo na ausência de dor, podem alterar de maneira distinta a intensidade do sinal em suas vocalizações, dependendo do estímulo, duração e grau de estresse. Considerando-se os valores de intensidade de som normal para suínos em condição de conforto citados por Cordeiro (2012), de 70,41 dB, observou-se na presente pesquisa que todos os estímulos estressantes proporcionaram valores médios superiores a esse. A frequência de pitch foi superior nas vocalizações de suínos submetidos ao estresse por calor e à condição de conforto, não sendo estatisticamente diferentes entre si. Esses valores foram seguidos pelo estresse por sede e fome, distintos entre si. Desse modo, esse atributo pode contribuir para diferenciar a condição de estresse por sede da condição de conforto, e o estresse térmico da condição de fome, para os quais os demais atributos não foram eficientes. A frequência de pitch não apresentou tendência de modificação ao longo do período de avaliação (Tab. 3 e Fig. 6).

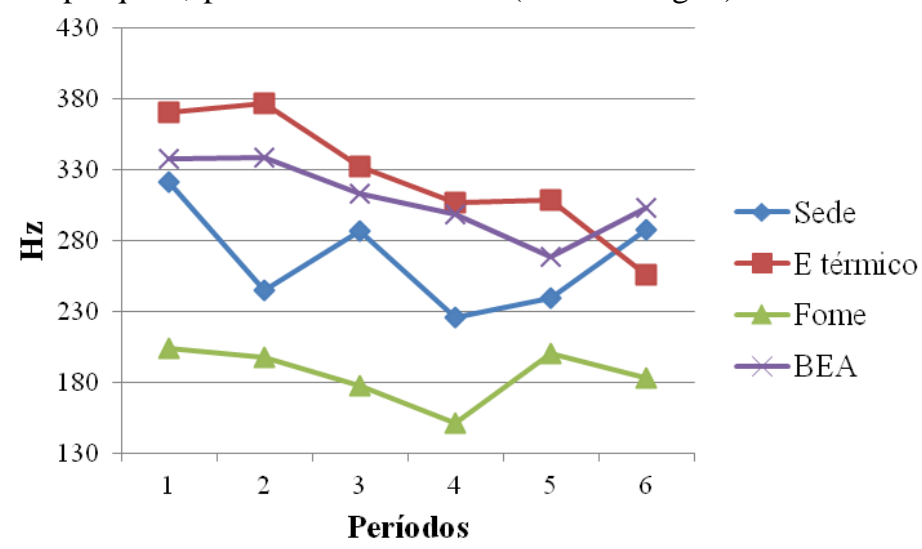

Figura 6. Frequência de pitch de vocalizações de suínos submetidos a diferentes estímulos estressantes. 
Diferentemente dos resultados da presente pesquisa, Cordeiro (2012) observou que a frequência de pitch apresentou valores maiores com o aumento do grau de estresse nos suínos. As formantes 1 e 3 não se apresentaram diferentes entre os tratamentos (Tab. 2), bem como não apresentaram padrão regular de comportamento ao longo do tempo de duração

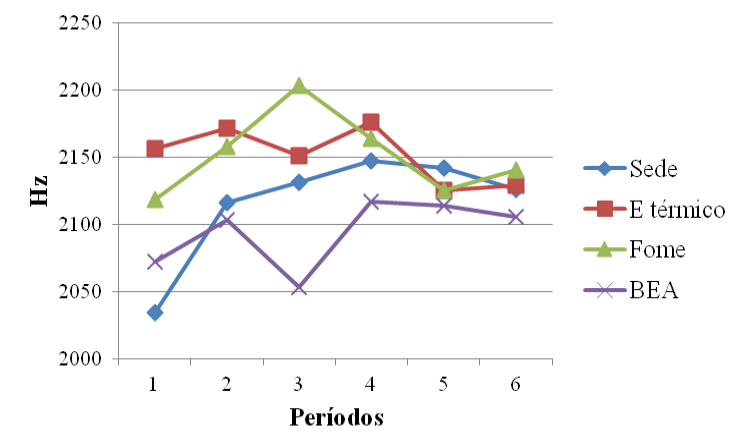

Figura 7. Formante 2 de vocalizações de suínos submetidos a diferentes estímulos estressantes.

Os valores de posição e dispersão das formantes (frequências harmônicas) oriundas da vocalização de animais sadios e com artrite apresentam diferenças significativas entre si $(\mathrm{P}<0,05), \mathrm{F} 1$ : sadios $1.287 \mathrm{~Hz}$ e com artrite 1.146 Hz; F2: sadios $2.430 \mathrm{~Hz}$ e com artrite $2.197 \mathrm{~Hz}$; F3: sadios $3.226 \mathrm{~Hz}$ e com artrite $3.248 \mathrm{~Hz}$ e F4: sadios $3.875 \mathrm{~Hz}$ e com artrite 3.889 , fornecendonos informações de que o timbre dos animais varia nas duas condições estudadas (Risi, 2010).

A utilização dos valores médios das formantes presentes na vocalização de vacas em duas condições distintas revelou resultados satisfatórios, sendo então variáveis acústicas que permitem a diferenciação entre a vocalização emitida em situação de estro e a vocalização emitida momentos antes da alimentação em bovinos (Yeon, 2006). As médias dos valores presentes em cada formante da vocalização de felinos forneceram bons resultados na tentativa de diferenciar as vocalizações emitidas durante o período pré-alimentar e em situações agonísticas (expressam agressividade). As primeiras e segundas formantes ressonantes são parâmetros do estímulo (Tab. 3). As formantes 2 e 4 só permitiram a diferenciação entre o estresse por fome e a condição de bem-estar, sendo entre os demais atributos semelhantes entre si. Ambas as formantes atingiram valores máximos no $3^{\circ}$ período de avaliação, diminuindo novamente após esse período (Tab. 3, Fig. 7 e 8).

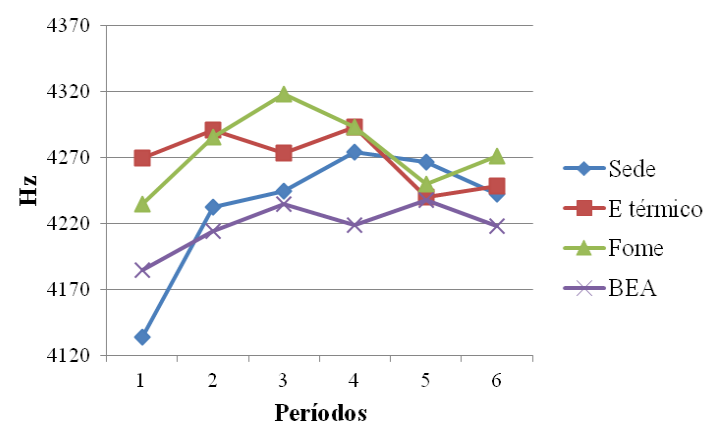

Figura 8. Formante 4 de vocalizações de suínos submetidos a diferentes estímulos estressantes.

acústicos importantes para diferenciar a vocalização de gatos domésticos e selvagens, podendo refletir a natureza de filtragem (amplificação do sinal) do trato vocal (Nicastro, 2004). Observaram-se correlações, positivas e negativas, entre os diferentes atributos do som de vocalizações de suínos submetidos a diferentes condições de estresse (Tab. 2).

Correlações positivas foram observadas entre a duração do estímulo por sede e as formantes 2, 3 e 4 (Tab. 4). Em contrapartida, quanto maior a duração do estresse por sede menor a energia do sinal. A energia do sinal relacionou-se positivamente com a amplitude máxima, amplitude mínima, intensidade do sinal e frequência de pitch, e foi negativamente correlacionada com as formantes 2,3 e 4 . As amplitudes máxima e mínima foram positivamente correlacionadas entre si e com a intensidade do sinal e frequência de pitch. A frequência de pitch foi positivamente correlacionada à intensidade do sinal e negativamente com as formantes 2,3 e 4 . 
Tabela 4. Correlações entre a duração do estímulo e atributos do som, para condição de estresse por sede, fome, térmico e situação de BEA

\begin{tabular}{|c|c|c|c|c|c|c|c|c|c|c|}
\hline & $\mathrm{DE}$ & ES & AA & AI & IS & FP & $\mathrm{F} 1$ & $\mathrm{~F} 2$ & F3 & $\mathrm{F} 4$ \\
\hline & \multicolumn{10}{|c|}{ Estresse por Sede } \\
\hline $\mathrm{DE}$ & 1,00 & $-0,42^{* *}$ & - & - & - & - & - & $0,63^{+++}$ & $0,61^{+++}$ & $0,61^{+++}$ \\
\hline ES & & 1,00 & $0,61^{+++}$ & $0,45^{++}$ & $0,95^{+++}$ & $0,50^{++}$ & - & $-0,57^{* *}$ & $-0,57^{* *}$ & $-0,56^{* *}$ \\
\hline AA & & & 1,00 & $0,88^{+++}$ & $0,63^{+++}$ & $0,40^{++}$ & - & - & - & - \\
\hline AI & & & & 1,00 & $0,55^{++}$ & $0,46^{++}$ & - & - & - & - \\
\hline IS & & & & & 1,00 & $0,56^{++}$ & - & $-0,44^{* *}$ & $-0,44^{* *}$ & $-0,44^{* *}$ \\
\hline FP & & & & & & 1,00 & - & $-0,46^{* *}$ & $-0,40^{--}$ & $-0,51^{* *}$ \\
\hline $\mathrm{F} 1$ & & & & & & & 1,00 & $0,43^{++}$ & $0,39^{++}$ & $0,44_{++}$ \\
\hline F2 & & & & & & & & 1,00 & $0,94^{+++}$ & $0,96^{+++}$ \\
\hline F3 & & & & & & & & & 1,00 & $0,95^{+++}$ \\
\hline \multirow[t]{2}{*}{$\mathrm{F} 4$} & & & & & & & & & & 1,00 \\
\hline & \multicolumn{10}{|c|}{ Estresse por Fome } \\
\hline $\mathrm{DE}$ & 1,00 & - & - & - & - & - & - & - & - & - \\
\hline ES & & 1,00 & $0,58^{++}$ & $0,62^{+++}$ & $0,95^{+++}$ & - & - & - & - & - \\
\hline AA & & & 1,00 & $0,75^{+++}$ & $0,55^{++}$ & $0,44^{++}$ & - & - & $-0,39^{* *}$ & $-0,48^{* * *}$ \\
\hline AI & & & & 1,00 & $0,58^{++}$ & $0,39^{++}$ & - & $-0,43^{* *}$ & $-0,51^{* *}$ & $-0,57^{* *}$ \\
\hline IS & & & & & 1,00 & - & - & - & - & - \\
\hline FP & & & & & & 1,00 & - & - & $-0,37^{* *}$ & $-0,49^{* *}$ \\
\hline F1 & & & & & & & 1,00 & - & - & - \\
\hline F2 & & & & & & & & 1,00 & $0,88^{+++}$ & $0,82^{+++}$ \\
\hline F3 & & & & & & & & & 1,00 & $0,95^{+++}$ \\
\hline \multirow[t]{2}{*}{$\mathrm{F} 4$} & & & & & & & & & & 1,00 \\
\hline & \multicolumn{10}{|c|}{ Estresse Térmico } \\
\hline $\mathrm{DE}$ & 1,00 & $-0,42^{* * *}$ & - & - & $-0,44^{* * *}$ & $-0,61^{* * * *}$ & - & $-0,38^{* *}$ & $-0,40^{* *}$ & $-0,40^{* *}$ \\
\hline ES & & 1,00 & - & - & $0,98^{+++}$ & $0,59^{++}$ & - & - & - & - \\
\hline AA & & & 1,00 & $0,63^{+++}$ & - & - & - & - & - & - \\
\hline AI & & & & 1,00 & - & - & - & - & - & - \\
\hline IS & & & & & 1,00 & $0,65^{+++}$ & - & - & - & - \\
\hline FP & & & & & & 1,00 & - & - & - & - \\
\hline $\mathrm{F} 1$ & & & & & & & 1,00 & $0,71^{+++}$ & $0,62^{+++}$ & $0,60^{++}$ \\
\hline $\mathrm{F} 2$ & & & & & & & & 1,00 & $0,92^{+++}$ & $0,89^{+++}$ \\
\hline F3 & & & & & & & & & 1,00 & $0,95^{+++}$ \\
\hline \multirow[t]{2}{*}{$\mathrm{F} 4$} & & & & & & & & & & 1,00 \\
\hline & \multicolumn{10}{|c|}{ Situação de BEA } \\
\hline $\mathrm{DE}$ & 1,00 & - & $-0,41^{* * *}$ & $-0,37^{* *}$ & $-0,47^{* *}$ & $-0,54^{* *}$ & - & - & - & - \\
\hline ES & & 1,00 & $0,77^{+++}$ & $0,76^{+++}$ & $0,79^{+++}$ & $0,42^{++}$ & - & - & - & - \\
\hline AA & & & 1,00 & $0,90^{+++}$ & $0,83^{+++}$ & $0,59^{++}$ & - & - & - & - \\
\hline $\mathrm{AI}$ & & & & 1,00 & $0,85^{+++}$ & $0,44^{++}$ & - & - & - & - \\
\hline IS & & & & & 1,00 & $0,53^{++}$ & - & - & - & - \\
\hline FP & & & & & & 1,00 & - & - & - & - \\
\hline $\mathrm{F} 1$ & & & & & & & 1,00 & - & $0,38^{++}$ & - \\
\hline F2 & & & & & & & & 1,00 & - & - \\
\hline F3 & & & & & & & & & 1,00 & $0,66^{+++}$ \\
\hline $\mathrm{F} 4$ & & & & & & & & & & 1,00 \\
\hline
\end{tabular}

+ - Correlação positiva fraca $(0,1$ a 0,3$)$; ++ - Correlação positiva moderada $(0,31$ a 0,6$)$; +++ - Correlação positiva forte $(0,61$ a 1,0$)$. * - Correlação negativa fraca $(0,1$ a 0,3$)$; ** - Correlação negativa moderada $(0,31$ a 0,6$)$; *** Correlação negativa forte $(0,61$ a 1,0). DE: duração do estímulo; ES: energia do sinal; AA: amplitude máxima; AI: amplitude mínima; IS: intensidade do sinal; FP: frequência de pitch; F1: formante 1; F2: formante 2; F3: formante 3; F4: formante 4 .

De maneira distinta ao observado para o estímulo sede, a duração do estímulo por fome não apresentou correlação com nenhum dos atributos de som avaliados. A energia do sinal comportouse de maneira semelhante ao estresse por sede, sendo positivamente correlacionada com as amplitudes máxima e mínima e alta correlação com a intensidade do sinal, mas, de maneira distinta, não se correlacionou com a frequência de pitch nem com as formantes. As amplitudes 
máxima e mínima foram positivamente correlacionadas entre si e com a intensidade do sinal e frequência de pitch, mas negativamente correlacionadas com as formantes. Correlações negativas foram observadas entre a frequência de pitch e as formantes 3 e 4 .

A duração do estímulo por estresse térmico correlacionou-se negativamente com a energia e intensidade do sinal, com a frequência de picth e com as formantes 2, 3, e 4, apresentando, dessa forma, padrão bastante distinto dos estímulos de fome e sede. Moderada correlação positiva foi observada entre a energia do sinal e a intensidade do sinal e frequência de picth, não apresentando correlação com as amplitudes máxima e mínima, conforme observado para os estímulos de fome e sede. Amplitudes máxima e mínima também apresentaram alta correlação positiva entre si, mas não se correlacionaram com nenhum outro parâmetro avaliado. Houve alta correlação positiva entre a intensidade do sinal e a frequência de pitch.

As correlações entre os atributos sonoros em condição de bem-estar assumiram padrão mais semelhante ao observado no estresse por sede, exceto pelo fato de as formantes praticamente não terem se correlacionado com os demais atributos e correlações negativas observadas entre a duração do estímulo e as amplitudes máxima e mínima, intensidade do som e frequência de pitch. As formantes foram alta e positivamente correlacionadas entre si em todas as situações de estresse, mas de maneira menos expressiva para a condição de BEA.

\section{CONCLUSÃO}

Os atributos sonoros da vocalização de suínos variam de maneira distinta em função do tipo e duração do estímulo estressante, funcionando como ferramenta eficiente para quantificar o grau de estresse dos animais, desde que associados uns aos outros.

\section{REFERÊNCIAS}

ASSOCIAÇÃO BRASILEIRA DA INDÚSTRIA PRODUTORA E EXPORTADORA DE CARNE SUÍNA - ABIPECS. Estatísticas. Disponível em: <http://www.abipecs.org.br>. Acessado em: $10 / 06 / 2015$.
BARBARI, M.; BIANCHI, M.; GUERRI, F.S. Preliminary analysis of different cooling systems of sows in farrowing room. J. Agr. Eng., v.1, p.9-15, 2007.

CORDEIRO, A. F. S. Uso da vocalização como ferramenta para identificação de suínos, medida de bem estar e estimativa da energia gasta na sua vocalização. 2012. nf. Tese (Doutorado em Engenharia Agrícola), Universidade Estadual de Campinas, Campinas, SP.

CORDEIRO, A. F. S.; PEREIRA, E. M.; NÄÄS. I. A. et al. Medida de vocalização de suínos (Sus scrofa) como um indicador de gasto energético. Rev. Bras. Eng. Biosist., v.2, p.1-5, 2009.

DÜPJAN, S.; SCHÖN, P. PUPPE, B. et al. Differential vocal responses to physical and mental stressors in domestic pigs (Sus scrofa). Appl. Anim. Behav. Sci., v.114, p.105-115, 2008.

GOYMANN, W.; EAST, M. L.; WACHTER, B. et al. Social status does not predict corticosteroid levels in postdispersal male spotted hyenas. Horm. Behav., v.43, p.474-479, 2003.

HESSING, M.J.C.; HAGELSO, A.M.; VAN BEEK, J.A.M. et al. Individual behavioural characteristics in pigs. Appl. Anim. Behav. Sci., v. 37, p. 285-295, 1993.

HÖTZEL, M. J.; MACHADO FILHO, L. C. P. Bemestar animal na agricultura do século XXI. Rev. Etol., v.6, p.3-16, 2004.

MARX, G.; HORN, T.; THIELEBEIN, J. et al. Analysis of pain-related vocalization in young pigs. $J$. Sound Vib., v.266, p.687-698, 2003.

NICASTRO, N. Perceptual and acoustic evidence for species-Level differences in meow vocalizations by domestic cats (Felis catus) and african wild cats (Felis silvestris lybica). J. Comp. Psychol., v.118, p.287-296, 2004.

RISI, N. Uso da vocalização como indicador patológico em leitões na fase de maternidade. 2010. 93f. Dissertação (Mestrado) - Escola Superior de Agricultura "Luiz de Queiroz", Piracicaba, SP.

ROLLER, W.L.; GOLDMAN, R.F. Response of swine to acute heat exposure. Trans. ASAE, v.12, n.2, p.164-169, 174, 1969.

RORIZ, M. Psicrom 1.0 - Relações psicométricas. São Carlos: Universidade Federal de São Carlos, 2003.

STATISTICAL Analysis System - SAS 9.2. Cary, NC: SAS Institute, 2000.

YEON, S.C.; JEON, J.H.; HOUPT, K. A. et al. Acoustic features of vocalizations of Korean native cows (Bos Taurus coreanea) in two different conditions. Appl. Anim. Behav. Sci., v.101, p.1-9, 2006. 